\section{Avaliação da conformidade do transporte neonatal para hospital de referência do Ceará}

\section{An evaluation of the extent to which the transportation of newborns to a referral hospital conforms to existing norms in the Brazilian State of Ceará}

\begin{abstract}
Objectives: to evaluate the conformity of the transport of newborns to existing norms in the State of Ceará.

Methods: a normative evaluation was carried out covering newborns transported to a neonatal referral unit in Fortaleza-CE. The extent to which transportation conformed to existing norms was evaluated, including issues regarding pre-transportation communication, the characteristics of the team and the clinical conditions on the arrival of the newborns and classified as: a) standard transportation; b) minimal transportation conditions; c) inadequate transportation. An arithmetic mean was calculated for each neonatal transportation based on the percentages obtained from the evaluated items and divided into three terciles representing high, average and low conformity.

Results: the study covered the transportation of 75 newborns with a mean weight and gestational age of $2121 \mathrm{~g}$ and 34.3 weeks respectively. The mean age on admission to hospital was 64.7 hours. The transportation of newborns was beset by serious deficiencies, with clear failures to meet the existing norms. Around $70 \%$ of newborns were transported in an inadequate manner. On no occasion was a newborn transported in full accordance with the norms. There was more conformity with the norms in the case of pre-transportation communication, the characteristics of the team, and the transportation unit.

Conclusions: the transportation of newborns showed deficiencies in the following respects: pretransportation communication, the adequacy of the team and the transport unit and clinical conditions on arrival.
\end{abstract}

Key words Health services evaluation, Intensive care units, neonatal, Transportation of patients
Ana Marita Araújo de Albuquerque 1

Álvaro Jorge Madeiro Leite 2

Nádia Maria Girão Saraiva de Almeida 3

Cristiana Ferreira da Silva 4

${ }^{1}$ Hospital Distrital Gonzaga Mota - Barra do Ceará. Fortaleza, CE, Brasil.

2 Departamento de Saúde Materno Infantil. Faculdade de Medicina. Universidade Federal do Ceará. Fortaleza, CE, Brasil.

3 Escola de Saúde Pública do Ceará. Fortaleza, CE, Brasil.

4 Núcleo Hospitalar de Epidemiologia. Hospital Municipal de Maracanaú Dr. João Elísio de Holanda. Rua João de Alencar, s.n. Centro. Maracanaú, CE, Brasil. CEP: 60.900-150.

E-mail: silva_ferreira_cristiana@yahoo.com.br

\title{
Resumo
}

Objetivos: avaliar a conformidade do transporte neonatal prestado a recém-nascidos no Estado do Ceará.

Métodos: pesquisa avaliativa normativa. Foram estudados recém-nascidos transportados até uma unidade neonatal de referência de Fortaleza-CE. Avaliou-se a conformidade dos transportes neonatais às normas técnicas em vigência, abordando aspectos da comunicação pré-transporte, características da equipe e unidade de transporte e condições clínicas de chegada dos recém-nascidos transportados e classificando-os em: a) transporte padrão; b) condições minimas de transporte; c) transporte inadequado. Cada transporte neonatal avaliado foi representado por média aritmética calculada com base nos percentuais obtidos dos itens avaliados e distribuidas em tercis, classificando-os em alta, média ou baixa conformidade.

Resultados: foram analisados os transportes de 75 recém-nascidos com médias de peso e de idade gestacional de $2121 \mathrm{~g}$ e 34,3 semanas respectivamente. A idade média por ocasião da admissão foi de 64,7 horas de vida. Os transportes neonatais realizados apresentaram deficiências graves, com nítido descumprimento das normas vigentes. Cerca de $70 \%$ dos recém-nascidos foram transportados de forma inadequada. Nenhum transporte neonatal foi considerado padrão. Maiores proporções de conformidade foram observadas nos aspectos referentes à comunicação pré-transporte, características da equipe e unidade de transporte.

Conclusões: os transportes neonatais apresentaram deficiências nos aspectos: comunicação prétransporte, adequação da equipe e unidade de transporte e condições clínicas de chegada.

Palavras-chave Avaliação de Serviços de Saúde, Unidades de Terapia Intensiva Neonatal, Transporte de pacientes 


\section{Introdução}

$\mathrm{O}$ transporte inter-institucional refere-se à transferência de pacientes entre unidades hospitalares ou não, de caráter público ou privado, que funcionem como base para a estabilização de pacientes graves. ${ }^{1}$

Em relação ao transporte do recém-nascido, a transferência de um prematuro para um hospital de referência foi descrita em 1900 por Segal, inaugurando a era moderna do transporte neonatal. ${ }^{2}$

A avaliação da conformidade do transporte neonatal representa um importante aspecto da assistência perinatal, considerando elementos essenciais para o sistema de transferência neonatal tais que: organização, comunicação, pessoal, equipamentos e unidades de transporte, bem como o sistema regionalizado e hierarquizado de atenção perinatal. A capacidade de manejo clínico, adequação da assistência ao recém-nascido durante o nascimento e seu transporte contribuem para o alcance de desfechos positivos em unidades neonatais de referência. 2,3

As deficiências nos serviços de transporte neonatal têm sido descritas em todo mundo. Nos países desenvolvidos inclusive a qualidade do transporte neonatal é objeto de estudo de avaliação de serviços de saúde e entre aqueles em desenvolvimento o interesse pelo transporte neonatal é recente, porém crescente. ${ }^{3-7}$ As condições necessárias para oferecer um transporte neonatal de qualidade já estão bem estabelecidas na literatura, $1,8-10$ porém vários estudos descrevem situações graves decorrentes do descumprimento às normas. $3-6$

A avaliação da conformidade do sistema de transporte neonatal e, por conseguinte, o conhecimento de suas deficiências se constituem em uma ferramenta importante para o aprimoramento dos serviços de neonatologia, propiciando a otimização dos recursos e determinando impactos na morbidade e mortalidades neonatais. ${ }^{11}$ Apesar da relevância do tema ainda existem poucos estudos no Brasil.

O presente estudo foi desenvolvido com o objetivo de avaliar a conformidade do transporte neonatal prestado aos recém-nascidos no Estado do Ceará, considerando as normas vigentes.

\section{Métodos}

Foi realizada uma pesquisa avaliativa normativa do transporte neonatal oferecido aos recém-nascidos transferidos de unidades de saúde no Estado do Ceará e admitidos na Unidade de Terapia Intensiva Neonatal (UTIN) do Hospital Geral Dr. Waldemar Alcântara (HGWA), localizada na cidade de
Fortaleza, capital do Estado.

Foram avaliados todos os recém-nascidos admitidos na UTIN do HGWA no período de julho a dezembro de 2004, tendo sido excluídos os recémnascidos que chegaram sem vida à unidade de referência, os provenientes de outro Estado Federativo, os oriundos de seus domicílios, os transportados sem responsabilidade de qualquer unidade de saúde ou equipe de saúde e aqueles encaminhados para unidade neonatal por necessidade de cuidados intermediários.

A pesquisa considerou a análise de documentos normativos, relatórios técnicos e legislação a respeito das condições padrão de transporte neonatal no âmbito nacional e estadual. Apoiados na literatura especia-lizada foram construídos indicadores de avaliação de conformidades à legislação e normas técnicas vigentes a partir de questionários que contemplassem as recomendações sobre os padrões de refe-rência estabelecidos pelo Conselho Federal de Medicina e pelas portarias do Ministério da Saúde. 1,8-10,12

A fonte de dados da pesquisa incluiu a observação direta (por ocasião da admissão), entrevistas estruturadas (com a mãe e familiares do recémnascido) e análise documental (informações contidas no relatório de transferência, prontuário, dados do hospital de origem)

A coleta de dados foi realizada por meio de questionário estruturado e aplicado pela pesquisadora (AMAA) e pelos demais médicos plantonistas do corpo clínico da UTIN. Ocorreu em dois momentos: a) por ocasião da solicitação de vaga; b) chegada dos pacientes no hospital de referência.

Foram construídos três grupos de conformidades por meio dos quais foram mensurados os serviços de transporte neonatal nos seguintes aspectos: comunicação pré-transporte, composição da equipe responsável pelo transporte, da unidade de transporte e do equipamento disponível na unidade e das condições clínicas dos recém-nascidos no momento da chegada: Grupo I - Comunicação pré-transporte, abordando a existência de contato prévio da Central Reguladora de Leitos do Município, a presença e o preenchimento dos relatórios de transferência; Grupo II - Composição da equipe responsável pelo transporte, da Unidade de transporte e do equipamento disponível na unidade; Grupo III - Condições clínicas dos recém-nascidos no momento da chegada: presença de distermias, alteração de glicemia capilar, venóclise funcionante, sinal indireto de hipoxemia pela oximetria de pulso e necessidade de progressão de suporte respiratório dentro da primeira hora após a admissão (Tabela 1). A 
construção dos grupos de conformidades e a análise dos resultados obtidos basearam-se na adaptação de metodologia semelhante utilizada por Eduardo e Novaes ${ }^{13}$ em estudo sobre conformidades às normas técnicas de proteção radiológica dos serviços de radioterapia no Estado de São Paulo.

Cada grupo foi composto por itens de conformidades e estes por perguntas, com escores para cada uma delas variando de 0 a 2 , indicando o não cumprimento (0), o cumprimento parcial (1) e o cumprimento total (2) daquele quesito. No caso do Grupo III, os escores variaram apenas entre 0 e 2, indicando apenas a presença ou ausência da condição mórbida.

O percentual de conformidade por grupo em cada um dos transportes avaliados foi obtido por meio da soma dos escores das perguntas, transformada em percentual em relação aos pontos brutos máximos possíveis especificados para cada grupo (Grupos I, II e III). Como cada grupo tem números diversos de itens, não se mostrou pertinente utilizar apenas o total de pontos obtidos.

Cada transporte neonatal avaliado foi representado por uma média aritmética calculada com base nos percentuais alcançados nos respectivos itens avaliados para cada grupo específico. As médias obtidas em cada um dos 75 transportes avaliados foram distribuídas em tercis, caracterizando-se os transportes em alta, média ou baixa conformidade.

Considerando que os quesitos utilizados como indicadores de avaliação dentro de cada grupo, possuem pesos diferentes na determinação dos resultados, foram determinados itens indispensáveis (equipe de transporte adequada, incubadora de transporte, material básico de reanimação, venóclise funcionante, oxigênio contínuo e adrenalina) que deveriam ser contemplados em todos os transportes, sob o risco de seu descumprimento acarretar graves consequências aos pacientes transportados.

Os transportes neonatais foram classificados em: a) Transporte padrão, quando existiu comunicação pré-transporte, relatório completo apresentado à admissão equipe de transporte completa, ambulância tipo D (tipo UTI), material básico de reanimação completo (ressuscitador manual, máscaras, laringoscópio e cânulas traqueais de tamanhos variados), medicações de urgência, venóclise funcionante, oxigênio contínuo durante toda a viagem; b) Condições mínimas de transporte, com equipe de transporte completa, incubadora de transporte, mate-

Tabela 1

Descrição das variáveis, escores brutos e valores percentuais alcançados segundo os grupos de conformidade do transporte neonatal. Hospital Geral Dr. Waldemar Alcântara, Fortaleza-CE, 2004.

\begin{tabular}{|c|c|c|}
\hline Grupos e itens de conformidade do transporte neonatal & Escores brutos máximos & Alcance (\%) \\
\hline Grupo I - Comunicação pré-transporte & 10 & 100,0 \\
\hline Relatório de transferência & 2 & 20,0 \\
\hline História materna & 2 & 20,0 \\
\hline História das condições de parto & 2 & 20,0 \\
\hline Dados completos do recém-nascido & 2 & 20,0 \\
\hline Solicitação de vaga compatível com quadro clínico & 2 & 20,0 \\
\hline Grupo II - Equipe e unidade de transporte & 26 & 100,0 \\
\hline Equipe de transporte & 2 & 7,7 \\
\hline UTI móvel & 2 & 7,7 \\
\hline Incubadora de transporte & 2 & 7,7 \\
\hline Material básico de reanimação & 2 & 7,7 \\
\hline Medicações & 10 & 38,5 \\
\hline Material para punção venosa & 2 & 7,7 \\
\hline Equipamento para suporte ventilatório & 6 & 23,0 \\
\hline Grupo III - Condições clínicas na admissão & 12 & 100,0 \\
\hline Distermias & 2 & 16,6 \\
\hline Glicemia alterada & 2 & 16,6 \\
\hline Venóclise funcionante na admissão & 2 & 16,6 \\
\hline Oxigênio contínuo durante todo o transporte & 2 & 16,6 \\
\hline Mudança de suporte ventilatório & 2 & 16,6 \\
\hline Saturação de oxiemoglobina na admissão & 2 & 16,6 \\
\hline
\end{tabular}


rial básico de reanimação completo, venóclise funcionante no momento da admissão, oxigênio contínuo durante todo o transporte e adrenalina; c) Transporte inadequado, quando não preencheu pelo menos um dos critérios relacionados na caracterização das condições mínimas de transporte. O primeiro tercil abrangeu percentuais de adequação cujos valores variaram de $16,6 \%$ a $45,8 \%$; o segundo de $47,9 \%$ a $68,7 \%$ e o terceiro de $72,9 \%$ a $87,5 \%$.

Os transportes também foram classificados de acordo com a avaliação de conformidade: Baixa conformidade (transportes neonatais cujas médias aritméticas calculadas com base nos percentuais de adequação obtidos nos respectivos itens avaliados para cada grupo específico, encontram-se distribuídas no primeiro tercil); Média conformidade (médias distribuídas no segundo tercil) e Alta conformidade (médias distribuídas no terceiro tercil).

O estudo foi aprovado pelo Comitê de Ética em Pesquisa Clínica do HGWA e recebeu o consentimento dos responsáveis pelos recém-nascidos.

\section{Resultados}

Foram estudados os transportes de 75 recémnascidos, sendo 43 do sexo masculino $(57,3 \%)$. A média do peso ao nascer foi de $2121 \mathrm{~g}( \pm 985,7 \mathrm{~g}) \mathrm{e}$ idade gestacional de $34,3( \pm 3,8)$ semanas. A idade média por ocasião da admissão foi de 64,7 horas de vida.

Os principais diagnósticos que motivaram a transferência foram: prematuridade $(61,3 \%)$, síndrome do desconforto respiratório $(38,7 \%)$, síndrome de aspiração meconial em 37,4\% e asfixia perinatal diagnosticada pelo quadro clínico e laboratorial compatível após admissão na unidade neonatal em $34,7 \%$.

Os recém-nascidos eram procedentes da capital do Ceará em $62,7 \%$ dos casos, sendo encaminhados principalmente de unidades de saúde secundárias $(61,3 \%)$, localizadas a menos de $50 \mathrm{~km}$ do hospital de estudo (76\%). A média de duração dos transportes neonatais foi de 61 minutos, alcançando tempo máximo de cinco horas. Dos pacientes estudados, $12 \%$ passaram por mais de um serviço antes da admissão na unidade de referência.

Apesar de todos os pacientes necessitarem de cuidados intensivos, foram transportados por profissional não habilitado para tal função em $42,7 \%$ das situações. Em 56\% dos casos a ambulância não se enquadrava em nenhuma especificação de conformidade.

Os itens básicos para reanimação neonatal estavam presentes no veículo de transporte no momento da admissão e disponível durante o transporte em $74,7 \%$ dos casos. Em nenhum transporte o respirador neonatal esteve presente. Cilindros de ar comprimido foram observados em apenas $5,3 \%$ dos casos. Apesar da presença do cilindro de oxigênio na unidade de transporte em $97,3 \%$ dos casos, em cinco situações $(6,7 \%)$ a quantidade do gás não foi suficiente para cobrir todo o percurso, esgotando-se antes da admissão na unidade de referência. A adrenalina não estava presente em $72 \%$. O fenobarbital esteve presente em apenas $20 \%$ dos transportes, ainda que $35 \%$ dos pacientes fossem considerados asfixiados graves nos relatórios de transferência.

A comunicação pré-transporte foi realizada pela Central Reguladora de Leitos do Município em praticamente todos os casos $(97,3 \%)$. Houve boa regulação dos leitos disponíveis, com avanço na identificação dos recém-nascidos de risco através de triagem efetiva, priorizando pacientes em estado mais crítico. Em apenas $25,3 \%$ das situações, o relatório de transferência continha todas as informações necessárias solicitadas pelo hospital de referência.

Com relação às condições clínicas no momento da admissão, constatou-se que $48 \%$ dos recémnascidos apresentavam-se distérmicos, sendo mais comum a presença de hipotermia $(45,3 \%)$. Os valores de Destrostix variaram entre 25 e 328, com média de $101 \mathrm{mg} / \mathrm{dL}$ e em cerca de $30 \%$ dos pacientes estavam alterados no momento da admissão hospitalar. Apenas $28 \%$ dos pacientes apresentaram saturação de oxigênio dentro dos limites considerados ideais (entre 89 e 95\%). A necessidade de progressão do suporte respiratório para ventilação mecânica e pressão positiva contínua (CPAP) na primeira hora após a admissão na unidade neonatal ocorreu em $32 \%$ dos transportes realizados (Tabela 2).

Nenhum dos transportes avaliados foi considerado padrão, sendo que em apenas $30 \%$ das transferências foram observados os critérios mínimos preconizados. O percentual de conformidade para cada grupo também foi baixo. Maiores proporções de conformidade foram observadas nos aspectos referentes à comunicação pré-transporte, características da equipe e unidade de transporte (Figuras $1 \mathrm{e}$ 2).

Verificou-se o predomino de transportes neonatais classificados como média conformidade $(46,7 \%)$, constatando-se que aproximadamente $30 \%$ foram considerados de baixa conformidade (Figura $3)$. 
Distribuição das variáveis relacionadas ao transporte neonatal segundo a comunicação pré-transporte, equipe e unidade de transporte neonatal e condições clínicas observadas na admissão hospitalar. Hospital Geral Dr. Waldemar Alcântara, Fortaleza-CE, 2004.

\begin{tabular}{|c|c|c|}
\hline Características do transporte neonatal & $N=75$ & $\%$ \\
\hline \multicolumn{3}{|l|}{ Comunicação pré-transporte } \\
\hline \multicolumn{3}{|l|}{ Presença de relatório de transferência } \\
\hline $\operatorname{Sim}$ & 74 & 98,7 \\
\hline Não & 1 & 1,3 \\
\hline \multicolumn{3}{|c|}{ Relatório sobre história materna e recém-nascido completo } \\
\hline Sim & 19 & 25,3 \\
\hline Não & 56 & 74,7 \\
\hline \multicolumn{3}{|c|}{ Solicitação de vaga compatível com quadro clínico na admissão } \\
\hline Sim & 70 & 93,3 \\
\hline Não & 5 & 6,7 \\
\hline \multicolumn{3}{|l|}{ Regulação pela central de leitos } \\
\hline Sim & 73 & 97,3 \\
\hline Não & 2 & 2,7 \\
\hline \multicolumn{3}{|l|}{ Equipe e unidade de transporte neonatal } \\
\hline \multicolumn{3}{|l|}{ Equipe de transporte } \\
\hline Pediatra qualificado & 43 & 57,3 \\
\hline Outro médico & 11 & 14,7 \\
\hline Profissional de nível médio & 21 & 28,0 \\
\hline \multicolumn{3}{|l|}{ Unidade de Terapia Intensiva móvel } \\
\hline $\operatorname{Sim}$ & 33 & 44,0 \\
\hline Não & 42 & 56,0 \\
\hline \multicolumn{3}{|l|}{ Incubadora de transporte } \\
\hline Sim & 41 & 54,7 \\
\hline Não & 34 & 45,3 \\
\hline \multicolumn{3}{|l|}{ Material básico de reanimação } \\
\hline Sim & 56 & 74,7 \\
\hline Não & 19 & 25,3 \\
\hline \multicolumn{3}{|l|}{ Equipamento para suporte ventilatório } \\
\hline $\operatorname{Sim}$ & 0 & - \\
\hline Não & 75 & 100,0 \\
\hline \multicolumn{3}{|c|}{ Condições clínicas observadas na admissão hospitalar } \\
\hline \multicolumn{3}{|c|}{ Distermias } \\
\hline Hipotermia & 34 & 45,3 \\
\hline Hipertermia & 2 & 2,7 \\
\hline Ausente & 39 & 52,0 \\
\hline \multicolumn{3}{|l|}{ Alteração glicemia } \\
\hline Hipotermia & 6 & 8,0 \\
\hline Hipertermia & 15 & 20,0 \\
\hline Ausente & 54 & 72,0 \\
\hline \multicolumn{3}{|l|}{ Venóclise funcionante na admissão } \\
\hline $\operatorname{Sim}$ & 48 & 64,0 \\
\hline Não & 27 & 36,0 \\
\hline \multicolumn{3}{|l|}{ Mudança de suporte ventilatório } \\
\hline $\operatorname{Sim}$ & 24 & 32,0 \\
\hline Não & 51 & 68,0 \\
\hline \multicolumn{3}{|c|}{ Saturação de oxihemoglobina na admissão } \\
\hline$<89$ & 18 & 24,0 \\
\hline $89-95$ & 21 & 28,0 \\
\hline$>95$ & 36 & 48,0 \\
\hline
\end{tabular}


Figura 1

Classificação dos transportes neonatais segundo a conformidade em relação às normas vigentes. Hospital Geral Dr. Waldemar Alcântara, Fortaleza-CE, 2004.

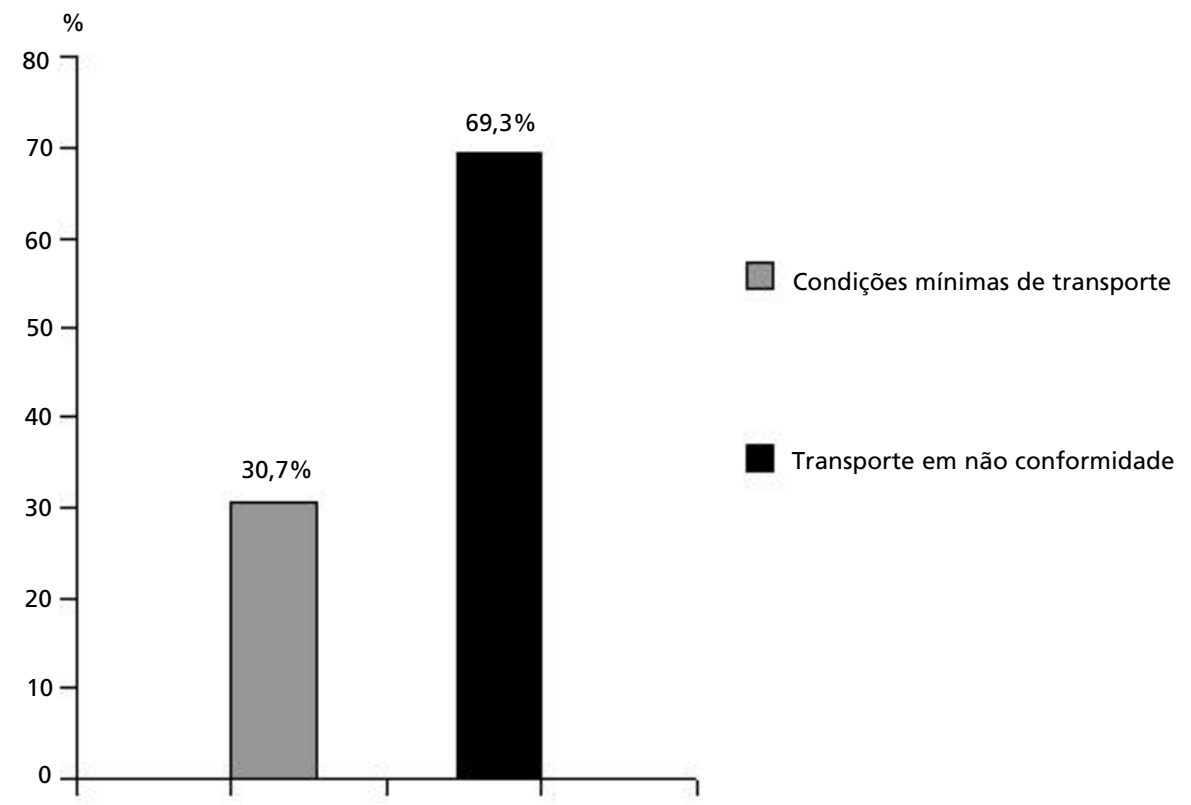

Figura 2

Proporção de conformidade por grupo: comunicação pré-transporte, composição da equipe responsável pelo transporte e condições clínicas dos recém-nascidos no momento da chegada. Hospital Geral Dr. Waldemar Alcântara, Fortaleza-CE, 2004.

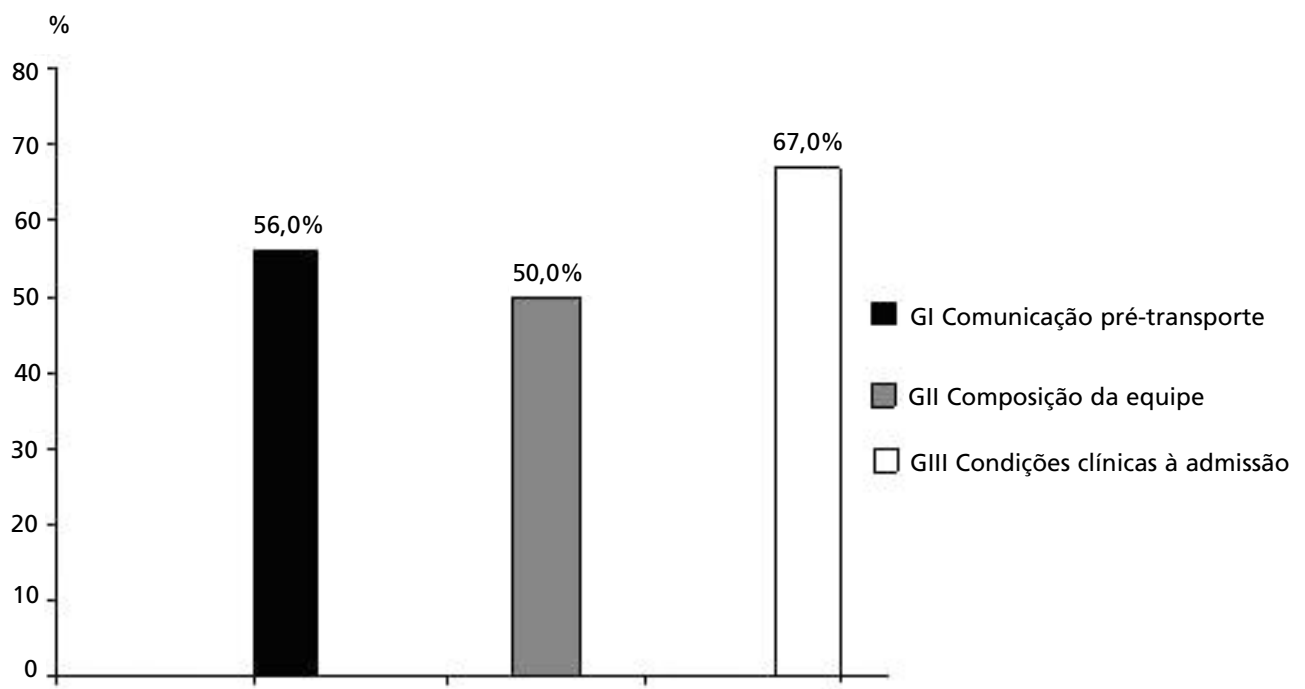


Classificação dos transportes neonatais segundo grau de conformidades Hospital Geral Dr. Waldemar Alcântara, Fortaleza-CE, 2004.

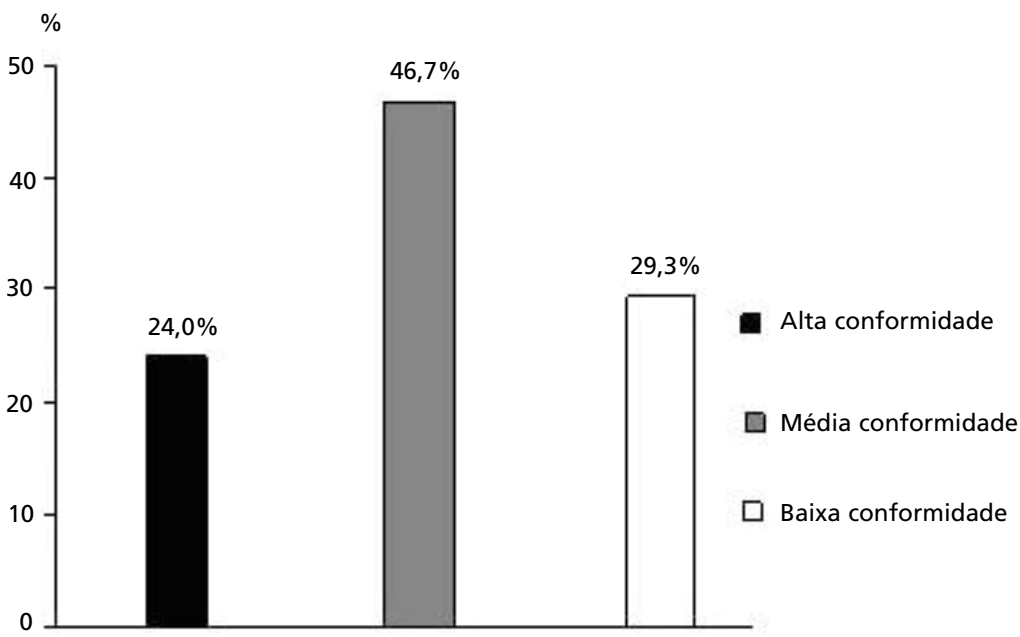

\section{Discussão}

A idade gestacional igual ou superior a 34 semanas admitidos na unidade de referência estudada sugere dificuldades em relação à organização da assistência perinatal regionalizada e com adequado poder de resolutividade das unidades secundárias de atendimento neonatal. 8

Em relação à equipe de transporte, a Agência Nacional de Vigilância Sanitária preconiza não apenas a presença de médico, mas exige a obrigatoriedade do título de especialista em medicina intensiva neonatal ou, no mínimo, experiência comprovada de pelo menos um ano na área. ${ }^{9}$ Os resultados mostraram que os recém-nascidos foram transportados por profissionais não habilitados. A equipe de transporte deve contar com pelo menos dois indivíduos, além do condutor do veículo e pelo menos um dos profissionais deve ser capaz de executar todos os procedimentos necessários para o cuidado de recém-nascidos criticamente doentes. ${ }^{9} \mathrm{O}$ transporte rea-lizado por equipe não treinada representa um risco de morte cerca de $60 \%$ maior em relação ao rea-lizado por equipe qualificada. 14

O tipo de unidade de transporte utilizado também é de fundamental importância. Em decorrência da vulnerabilidade do recém-nascido seu transporte deverá ser realizado em ambulância do tipo D ou UTI Móvel, contendo além de todo equipamento estabelecido para ambulância do tipo suporte avançado de vida específico para uso neonatal, incubadora de transporte e respirador neonatal. 9

$\mathrm{Na}$ presente casuística, a unidade de transporte utilizada foi ambulância em todos os casos. O transporte rodoviário, acessível universalmente e de baixo custo, deve ser reservado para transferências cuja distância seja inferior a 150 ou $200 \mathrm{~km} .15,16$

Os resultados da avaliação da conformidade do transporte neonatal mostraram que nenhuma das ambulâncias possuía respirador neonatal. No transporte de pacientes graves, principalmente recémnascidos prematuros com insuficiência respiratória, que necessitam de ventilação assistida, existem duas preocupações importantes: a concentração ideal de oxigênio e os efeitos da variação na aplicação da pressão e do volume durante a ventilação manual.17 Nas duas situações o respirador neonatal é fundamental. Elevadas concentrações de oxigênio após períodos de hipóxia, levam à formação de grande quantidade de radicais livres excedendo a capacidade antioxidante do prematuro e determinando lesão pulmonar difusa. Elevadas pressões parciais de oxigênio também contribuem para a ocorrência de retinopatia da prematuridade. ${ }^{18}$ A ausência de cilindros de ar comprimido nas unidades de transportes neonatais acarreta a oferta de oxigênio em níveis mais elevados que o necessário. A alta prevalência de recém-nascidos prematuros transferidos relaciona-se direta ou indiretamente com a elevada incidência de complicações posteriores de elevada morbidade relacionadas à presença de radicais livres. 17

Em cinco transportes de recém-nascidos, o 
volume de oxigênio não foi suficiente para garantir a oferta durante todo o trajeto, determinando hipóxia grave com parada cardiorespiratória detectadas na admissão em dois casos. A suspensão abrupta de oxigênio, principalmente em pacientes com insuficiência respiratória aguda, traz consequências graves ao recém-nascido, comprometendo não apenas a sua sobrevivência, como determinando a possibilidade de sequelas neurológicas permanentes. 19

A incubadora de transporte estava presente em pouco mais da metade dos transportes neonatais avaliados. Estudo ${ }^{5}$ realizado no Peru constatou que $60 \%$ dos pacientes foram transportados em berços comuns ou nos braços de profissionais de enfermagem. Em Recife, menos de 10\% dos recémnascidos utilizaram este equipamento. $20 \mathrm{O}$ Ministério da Saúde determina que todas as viaturas para transporte de recém-nascidos devem estar equipadas com incubadora. A incubadora de transporte é essencial durante o transporte porque permite a constante visualização do neonato, evita a perda de calor e as alterações metabólicas graves conseqüentes a hipotermia. 10

Ressalta-se a ausência frequente das medicações básicas utilizadas no atendimento de emergências no período neonatal. A adrenalina é uma medicação de urgência largamente utilizada em reanimação neonatal e o fenobarbital endovenoso é preconizado como primeira escolha para o controle de convulsões no período neonatal.21 A convulsão é condição mórbida intimamente relacionada à presença de asfixia perinatal,21 diagnóstico descrito nesse estudo em frequência considerável (35\%). Episódios convulsivos ocorrem em cerca de $20 \%$ a $50 \%$ dos recém-nascidos com encefalopatia hipóxicoisquêmica e as manifestações são de difícil controle, necessitando de terapêutica urgente a fim de evitar maiores danos cerebrais. ${ }^{19}$ Portanto, a ausência em $80 \%$ de drogas anticonvulsivantes na unidade de transporte neonatal compromete gravemente a instituição da terapêutica em tempo hábil.

A presença de relatórios incompletos compromete a excelência no atendimento, visto que a carência de informações retarda as medidas diagnósticas e terapêuticas cabíveis em cada situação. 22

Cerca de metade dos pacientes apresentou distermias à admissão. A hipotermia representa uma condição de risco para todos os recém-nascidos e principalmente para os prematuros, visto que pode desencadear hipoxemia e acidose, agravando a patologia de base. $23 \mathrm{O}$ aumento da incidência de hipotermia está claramente associado ao aumento da mortalidade neonatal.20,24

Os distúrbios da glicemia representam uma das complicações mais frequentes durante o transporte neonatal. ${ }^{25}$ Lesões cerebrais embora sejam mais comuns nos casos de hipoglicemia sintomática também podem ser observados na ausência de sintomas. Já a hiperglicemia leva ao aumento da osmolaridade sérica, podendo predispor à hemorragia intracraniana e à desidratação por diurese osmótica. ${ }^{26}$

É consenso a necessidade de acesso venoso a fim de manter adequada administração de glicose durante todo o transporte, bem como para proporcionar adequado manejo nas situações de emergência. ${ }^{15}$ A estabilização mínima do recémnascido requer, obrigatoriamente, a presença de acesso venoso adequadamente fixado para evitar a sua perda. 5,22

A saturação da hemoglobina no momento da chegada à unidade neonatal foi inferior a $89 \%$ ou superior a $95 \%$ em $72 \%$ das situações. A saturação de hemoglobina entre $89 \%$ e $95 \%$ corresponde a uma pressão arterial entre 50 e $70 \mathrm{mmHg}$, considerada ideal para o período neonatal. Pressões parciais de oxigênio abaixo ou acima desses valores não são isentas de riscos. 18

A progressão do suporte respiratório na primeira hora após a admissão na unidade neonatal ocorreu em $32 \%$ dos casos. Tal situação sugere falha grave na estabilização do paciente no hospital de origem e/ou durante o seu transporte.22 A estabilização respiratória reduz a morbimortalidade neonatal, em especial devido à elevada probabilidade de deterioração clínica se medidas básicas não forem adotadas.6,22

Em Santa Catarina, metade dos pacientes transportados necessitou de assistência ventilatória mecânica no momento de sua admissão na UTI, ou pela severidade da insuficiência respiratória ou pelo comprometimento do estado geral. ${ }^{6}$ Neste estudo cerca de $20 \%$ dos pacientes com insuficiência respiratória foram encaminhados sem oxigenioterapia, sendo que em apenas $6,6 \%$ dos casos o transporte ocorreu com via aérea definitiva.

Nenhum transporte foi considerado padrão e cerca de $70 \%$ não contemplaram as condições mínimas para que a estabilidade ventilatória, hemodinâmica e térmica fossem alcançadas. $5,27 \mathrm{O}$ transporte realizado em condições inadequadas é capaz de aumentar a mortalidade neonatal, a morbidade e do tempo de internação dos pacientes, comprometendo a eficácia dos cuidados intensivos recebidos. 20,25

Ainda que as unidades terciárias utilizem todo o aparato tecnológico disponível, a sobrevida e a presença de sequelas graves podem ser consequência 
de eventos ocorridos antes da admissão na unidade neonatal, seja pela precária assistência ao parto, ou pela qualidade do transporte oferecido. ${ }^{3}$

Foram identificados baixos percentuais de conformidade. $\mathrm{O}$ grupo que evidenciou maior percentual foi aquele referente às condições clínicas de chegada ao hospital. Condições clínicas regulares à admissão diante de baixos percentuais de não conformidade às normas provavelmente decorrem do perfil associado a menor vulnerabilidade biológica dos pacientes transportados avaliados, ainda que criticamente enfermos. 28,29

O grupo que apresentou pior conformidade foi o referente à equipe e unidade de transporte. É grave tal constatação visto que não é possível minimizar riscos com insuficiência de recursos humanos qualificado e infraestrutura para a condução de situações emergenciais. ${ }^{14-16}$

\section{Referências}

1. Brasil. Portaria GM/MS no 2048 , de 5 de novembro de 2002. Transferências e Transporte Inter-Hospitalar. Capitulo VI. [Acesso em 28 set 2011]. Disponível em: http://200.198.43.10:8080/ses/atos_normativos/legislacaosanitaria/estabelecimentode-saude/urgencia-e-emergencia/portaria_2048_B.pdf

2. Buser-Gills M, Whitfield JM. Transport neonatal. In: Closkey K, editor. Pediatric transport medicine. St. Louis: Mosby; 1994. p. 404-45.

3. Carmo FLM. Fatores de risco para mortalidade neonatal em hospital público terciário no município de Fortaleza Ceará. [dissertação]. Fortaleza: Universidade Estadual do Ceará; 2002.

4. Cassali MC, Briansó BA, Salas D. Transporte neonatal em Costa Rica. Acta Pediatr Costarric. 1998; 12: 15-20.

5. Quispe JAM. Estandares minimos de calidad en el transporte neonatal en el Hospital Nacional Daniel Alcides Carrion Callao Enero-Diciembre 2001 [monografia]. Peru, Lima: Facultad Medicina Humana; 2003.

6. Silva CV, Pereira LDC. Transporte neonatal em Santa Catarina. Arq Catarin Med. 1995; 24: 11-6.

7. Leslie A, Stephenson T. Neonatal transfers by advanced neonatal nurse practitioners and paediatric registrars. Arch Dis Child Fetal Neonatal Ed. 2003; 88: 509-12.

8. Sociedade Brasileira de Pediatria. Infraestrutura para atendimento integral ao recém-nascido. Documento Científico do Departamento de Neonatologia da Sociedade Brasileira de Pediatria. Aprovado em 21/11/2010. [Acesso em 28 set 2011]. Disponível em: http://www.sbp.com.br/pdfs/SBP-DC-Neo-Infraestruturaintegral 21 nov2010aprovado.pdf

9. Brasil. Secretaria de Vigilância Sanitária. Portaria $n^{\circ}$ 466/98. Requisitos operacionais para os serviços de tratamento intensivo móvel. [Acesso em 9 set 2003]. Disponível em: http://www.anvisa.gov.br/legis/portarias/466_98.htm
Observou-se que os transportes apresentavam baixa e média conformidade. Os transportes neonatais classificados como média conformidade apresentaram proporção de adequação às normas vigentes variando entre $47,9 \%$ a $68,7 \%$, não atingindo, em algumas situações, sequer a metade das exigências investigadas e preconizadas como normas pelas portarias sanitárias do Brasil e recomendações do Conselho Federal de Medicina.1,8-10,12 Portanto, os transportes neonatais apresentaram deficiências graves em todos os aspectos avaliados. Apesar do amplo conhecimento do que é necessário para oferecer um transporte neonatal seguro e de qualidade, as normas foram descumpridas. Urge a adoção de medidas que garantam um transporte seguro aos recém-nascidos, contribuindo para a redução da morbimortalidade neonatal.

10. Brasil. Conselho Federal de Medicina. Legislação $n^{\circ}$ 1672/03 de 9 de julho de 2003. Transporte inter-hospitalar de pacientes. [Acesso em 9 set 2003]. Disponível em: http://dtr2001.saude.gov.br/samu/legislacao/leg_res1672.ht $\mathrm{m}$

11. Cornette L. Contemporary neonatal transport: problems and solutions. Arch Dis Child Fetal Neonatal Ed. 2004; 89: 2124.

12. Brasil. Ministério da Saúde. Secretaria de Atenção a Saúde. Departamento de Ações Programáticas e Estratégicas. Manual de orientações sobre o transporte neonatal / Ministério da Saúde, Secretaria de Atenção a Saúde, Departamento de Ações Programáticas e Estratégicas. Brasília, DF; 2010. 40 p.

13. Eduardo MBP, Novaes HMD. Análise de conformidades às normas técnicas de proteção radiológica dos serviços de radioterapia no Estado de São Paulo, Brasil. Cad Saúde Pública. 2004; 20: S256-67.

14. Hood JL, Cross A, Hulka B, Lawson EE. Effectiveness of the neonatal transport team. Crit Care Med. 1983; 11: 41923.

15. Rossel K. Transporte neonatal. Pediatria al dia. 1998; 14 : 294-7.

16. Bocanegra EEC, Rodríguez TM. Signos vitales y saturación de oxigeno durante el transporte neonatal intrahospitalario en um servicio de cuidados intensivos neonatales. Bol Méd Hosp Infant Mex. 2001; 12: 854-61.

17. Almeida MFB, Guinsburg R. A reanimação do prematuro extremo em sala de parto: controvérsias. J Pediatr. 2005; 81: S3-15.

18. Tin W, Milligan DW, Pennefather P, Hey E. Pulse oximetry, severe retinopathy, and outcome at one year in babies of less than 28 weeks gestation. Arch Dis Child Fetal. 2001; 84: $106-10$ 
19. Aurora S, Snyder EY. Perinatal asphyxia. In: Cloherty JP, Eichenwald EC, Stark AR, editores. Manual of neonata care. USA: Lippincott Williams\& Wilkins; 2004. p.143-9.

20. Silveira SMM, Mello MJG, Vidal SA, Frias PG, Cattaneo A. Hypothermia on admission: a risk factor for death in newborns referred to the Pernambuco Institute of Mother and Child. J Trop Pediatr. 2003; 49: 115-20.

21. Ceccon MEJR. Interleucinas na encefalopatia hipóxicoisquêmica. J Pediatr. 2003; 79: 280-1.

22. Ruiz MTE, Aloy JF, Alix AG, Ribes AA, Bravo DB, Lorenzo JRF. Recomendaciones para el transporte perinatal. Comitê de Estándares de la Sociedad Española de Neonatologia. An Esp Pediatr. 2001; 55: 146-53.

23. Chatson K. Temperature Control. In: Cloherty JP, Eichenwald EC, Stark AR, editores. Manual of neonatal care. USA: Lippincott Williams\& Wilkins; 2004. p.147-50.

24. Sinclair JC. Management of the thermal environment. In Sinclair JC, editor. Effective care of the newborn infant. New York: Oxford University Press; 1993. p. 40-56.

25. Lagunas CA, Bello CIS, Cobian XMP, Hernández KR, Pasillas MV, Gonzáles FG. Complicaciones del recién nascido en el transporte neonatal y la participación de la enfermera. Rev Sanid Milit Mex. 1998; 52: 343-6.
26. Meneguel, JF, Guinsburg R. Hemorragia peri-intraventricular: fatores e conduta. Programa de Atualização em Neonatologia (PRORN). In: Procianoy RS, Leone CR, editores. Porto Alegre, RS: Artmed/Panamericana; 2003. p. 59-84.

27. Cid JLH, Álvarez AC, Macías CC. Recomendaciones de reanimación cardiopulmonar básica, avanzada y neonatal. Estabilización posresucitación y transporte. An Esp Pediatr. 2000; 52: 457-63.

28. Almeida MF, Novaes HMD, Alencar GP, Rodrigues LP. Mortalidade neonatal no Município de São Paulo: influência do baixo peso ao nascer e fatores sócio-demográficos e assistenciais. Rev Bras Epidemiol. 2002; 5: 93-107.

29. Vieira ALP, Guinsburg R, Santos AMN, Peres CA, Lora MI, Miyoshi MH. Transporte intra-hospitalar de pacientes internados em UTI neonatal: fatores de risco para intercorrências. Rev Paul Pediatr. 25: 240-6.

Recebido em 3 de janeiro de 2011

Versão final apresentada em 23 de fevereiro de 2012

Aprovado em 24 de fevereiro de 2012 